\title{
The effect of elastic therapeutic taping on lumbar extensor isokinetic performance
}

Harry J Knapman ${ }^{1}$, Tom Fallon ${ }^{1}$, Matthew O'Connor ${ }^{1}$, Lee A Titmus ${ }^{1}$,

Sherrie T Choy ${ }^{2}$, Claire Hornsby ${ }^{1}$, Jon F Marsden ${ }^{1}$ and Gary L Shum ${ }^{3}$

${ }^{1}$ School of Health Professions, Plymouth University, United Kingdom

${ }^{2}$ Livewell Southwest, NHS, Plymouth, United Kingdom

${ }^{3}$ Faculty of Sport \& Health Sciences, University of St Mark \& St John, United Kingdom

Corresponding author:

Dr Gary Shum PhD, MCSP

Faculty Director of Research \& Associate Professor

Faculty of Sport \& Health Sciences

University of St Mark \& St John

Derriford Road, Plymouth PL6 8BH

United Kingdom

Email: gshum@marjon.ac.uk

Tel: 01752636700 (Ext. 5310) 


\title{
The effect of elastic therapeutic taping on lumbar extensor isokinetic performance
}

\author{
Abstract \\ Objective: To investigate the effects of elastic therapeutic tape when applied \\ overlaying the lumbar extensors on different measures of muscle performance, \\ compared to a placebo taping technique and a no-tape control.
}

Relevance: Elastic therapeutic tape is frequently used as an adjunct to enhance athletic performance amongst athletes. However, limited research exists supporting its application on isokinetic performance of the lumbar extensor muscles.

Methods: A cross-sectional experimental study. 21 participants received three taping conditions in a randomised order: elastic therapeutic tape, a placebo tape and a notape control. Peak torque, the time taken to reach peak torque and peak velocity were measured using an isokinetic dynamometer.

Analysis: Friedman's test and post-hoc Wilcoxon signed-rank test were used to determine the statistical differences between the three taping conditions. Level of significance was set at 0.05 .

Results: A statistically significant improvement in peak lumbar extensor torque was observed when comparing elastic therapeutic tape with the no-tape control $(p<$ 0.05). However, there was no significant differences in time taken to reach peak torque and peak velocity $(p>0.05)$.

Conclusions: Results demonstrate that the application of elastic therapeutic tape overlaying the primary lumbar extensors significantly improves the maximal lumbar extension peak torque in healthy, asymptomatic adults. 


\section{Introduction}

Individuals who participate in recreational, amateur or professional level sports frequently seek contemporary techniques designed to enhance their athletic capabilities (Lins, et al., 2013). Application of elastic therapeutic tape is an increasingly popular adjunct used to enhance muscle performance. It has been proposed that when applied under tension, elastic therapeutic tape generates microconvolutions in the epidermis, lifting the skin and myofascia away from the tissues beneath (Kase, et al., 2003). Kase (2003) proposed that this movement aids blood flow and lymphatic drainage, which may increase oxygen allotment for the underlying tissues and influence contractile potential(Kase, et al., 2003). Furthermore, an amplification of afferent stimulation via cutaneous receptors is proposed to facilitate the neuromuscular response, resulting in enhanced muscular performance (Kase, et al., 2003; Macgregor, et al., 2005).

Research has indicated that when applied, elastic therapeutic tape can influence isokinetic measures of muscle function and athletic performance (Fratocchi, et al., 2013; Wong, et al., 2012). A recent study demonstrated significant improvements in the concentric and eccentric peak torque, following the application of elastic therapeutic tape overlaying the biceps brachii muscle (Fratocchi, et al., 2013). A significant reduction in the time to peak torque (Wong, et al., 2012) has been demonstrated from the application of elastic therapeutic tape on the quadriceps muscles in maximal concentric contraction, however, no significant improvement in maximal concentric torque in quadriceps have been found (Poon, et al., 2015; Wong, et al., 2012). Other studies have established a positive effect on muscle activation following the application of elastic therapeutic tape, whereby EMG readings have illustrated an earlier onset of activity in medial gastrocnemius, vastus medialis 
obliquus and the lower fibers of trapezius (Hsu, et al., 2009; Huang, et al., 2011; Slupik, et al., 2007).

Mostert-Wentzel et al. (2012) conducted a double-blinded randomised controlled trial investigating the short-term effects of elastic therapeutic tape the explosive gluteus maximus power of sixty male athletes by measuring the vertical jump height. The results demonstrated significant increases $(p<0.05)$ in vertical jump height following application and thirty minutes later(Mostert-Wentzel, et al., 2012). Additional studies have also demonstrated an increase in functional performance following the application of elastic therapeutic tape in healthy subjects and therefore it could be considered feasible that the application of elastic therapeutic tape may influence muscle performance and in particular, explosive muscle power (Aktas \& Baltaci, 2011; Huang, et al., 2011).

Maximal muscle power can be described as the capability of the neuromuscular system to create a voluntary contraction at maximum velocity (Wilmore, et al., 2008). Within a sporting context, activities such as sprinting, jumping, throwing and kicking demand high velocity movements combined with high force generation requiring maximal muscle power(Newton \& Kraemer, 1994). The effect of elastic therapeutic tape on the peak velocity of muscle contraction has not been investigated in previous studies. It may be that this component of the muscular contraction is largely responsible for an improvement in isokinetic and functional ability suggesting the importance of its, its inclusion within the muscle testing procedure of this study.

Despite being a technique regularly employed by elite athletes, no previous research has been conducted exploring the effects that elastic therapeutic tape has on the isokinetic muscle performance of the lumbar extensor when applied overlaying 
the lumbar extensor muscles. Functional magnetic resonance imaging and electromyography has demonstrated the significant role that the erector spinae muscle group has during extension exercises and athletic activities (De Ridder, et al., 2015; Sharp, et al., 2014). Additionally, training programs targeting the lumbar extensor muscles have previously demonstrated significant improvements in vertical jump height and sprint speed, supporting the significant role that these muscles play in physical performance (Imai, et al., 2014). With this in mind, further research investigating the effects of elastic therapeutic tape on lumbar extensor muscle performance is warranted. The contrasting results of different studies also suggest that the effect of elastic therapeutic tape may differ from muscle to muscle. The aim of this study was to investigate the effects of elastic therapeutic tape on the lumbar extensor muscles isokinetic performance, including peak torque, time to peak torque and peak velocity, in comparison to a placebo taping method and a no-tape control. It has been hypothesized that there is a significant increase in isokinetic muscle performance following the application of elastic therapeutic tape in comparison to notape and placebo taping methods. 


\section{Materials and Methods}

The methodology was a comparative, single group, cross-over design with repeated measures (Polgar \& Thomas, 2008).The independent variable was the taping technique, whilst the dependent variable was the isokinetic and isotonic muscle performance of the lumbar extensors including concentric lumbar extension peak torque, time taken to reach peak torque and peak velocity of lumbar extension.

Participant recruitment took place using an incidental sampling method, allowing the selection of the most easily accessible members of the target population(Hicks, 2004).

A sample of 22 healthy participants were recruited. The demographic characteristics of participants were presented in Table 1. Inclusion into the study required participants to be healthy, asymptomatic and aged between eighteen and fifty years old. Volunteers were excluded if they had a previous musculoskeletal disorder of the spine within the last three-months, had any other serious pathologies, had a symptomatic musculoskeletal condition, and were allergic to tape or similar adhesive materials. One participant was excluded from taking part in the study due to a current symptomatic musculoskeletal condition. Twenty one participants eventually took part in the study. Volunteers received a participant information sheet detailing the aims of the study and ethical information, highlighting that participants withheld the right to withdraw at any time. If volunteers were then willing to take part, written consent was obtained.

Prior to the study, ethical approval was obtained from a university ethics committee (Ref: PHT309_3). The order in which participants received a taping 
technique was randomised using a random number generator prior to data collection. In order to reduce observer bias, the assessors were blinded to the application of the taping procedure and participants required to wear dark colour clothing to ensure the taping method was not visible.

Standardised elastic therapeutic tape was used throughout the study, whilst a standardised non-elastic, self-adhesive dressing retention tape was used as a placebo (Hypafix®, BSN Medical, Germany). Inclinometer, tape measure, calculator, stopwatch and eyeliner pencils were used in order to assist the taping procedure.

Concentric lumbar extension peak torque at $60 \%$, time taken to reach peak torque and peak velocity was measured using an isokinetic dynamometer (Biodex System III, Biodex Medical Systems Inc., New York, USA), which can be considered the 'gold standard' apparatus for isokinetic muscle testing (Stark, et al., 2011). The Biodex System III has previously demonstrated a high level of validity and reliability when measuring both peak torque and peak velocity (Drouin, et al., 2004b).

Prior to testing, participants carried out a five-minute warm-up on a static cycle ergometer with the resistance set at level 2. Immediately following, skin preparation took place using an alcohol wipe to clean the back. Adopting a standardised seated position with $20^{\circ}$ of lumbar flexion, measured using an inclinometer, an eyeliner mark was placed on the participants skin overlaying the erector spinae muscles at the level of the 7th thoracic vertebrae and the posterior superior iliac spine. This distance was then measured in order to guide the required amount of tape using a tape measure. For elastic therapeutic tape, this distance was then divided by 1.2, indicating the length of tape required in order to ensure that it is stretched to $120 \%$ of its original length as recommended by its manufacturers(Kase, et al., 2003). The Placebo tape was cut to this length only. Both taping techniques 
adopted a standardized technique to the skin overlaying the erector spinae muscle group between the two previously mentioned landmarks (Figure 1). Throughout this process, participants were instructed to maintain eye contact with the wall in front of them whilst tape preparation took place behind them so as to maintain participant blinding. Taping was also carried out behind a curtain in order to ensure blinding of the assessors. The time taken to apply each taping technique was approximately two-minutes. For the no-tape control, two-minutes was allocated to mimic the time taken to apply the tape, in order to ensure a standardised testing procedure.

Following tape application, participants adopted the 'seated compressed' position within the isokinetic dynamometer in accordance with the manufacturer's recommendations (Figure 2) (Biodex medical systems Inc., 2006). In a randomised order, participants carried out muscle testing at two angular velocities. Peak torque and time taken to reach peak torque were measured at an angular velocity of $60 \% \mathrm{~s}$ for four repetitions, in keeping with concentric velocities used in previous studies(Mueller, et al., 2012). Peak velocity was measured by instructing participants to perform maximal concentric contractions as fast as possible against no resistance. For measurement of peak velocity, the isokinetic dynamometer was set at $360 \% \mathrm{~s}$ with a minimal resistance of $10 \mathrm{Nm}$ as indicated in previous investigations(Drouin, et al., 2004a). Participants were instructed to carry out one sub-maximal attempt at each velocity in order to gain a sense of the required movement. This was followed by four recorded maximal attempts, with thirty-second rest between repetitions. $A$ two-minute rest interval was allowed between testing velocities in order to avoid participant fatigue. This process was then repeated adopting the remaining taping conditions. Throughout data collection, researchers provided standardised 
instructions to the participants, with no form of encouragement in order to reduce a risk of bias.

Statistical Analysis

The statistical package SPSS 21.0 (Statistical Package for Social Sciences, IBM, New York) was utilized for all statistical analyses. Where power calculations were required, the $\mathrm{G}^{\star}$ Power 3.1 statistical power analyses package(Faul, et al., 2007) was used.

The maximum peak torque and velocity out of the four trials was determined and used for data analysis. A Kolmogorov-Smirnov test was carried out alongside a histogram in order to determine whether parametric assumptions had been met on the peak torque. The results of the Kolmogorov-Smirnov test suggested that there was a significant deviation from the normality $(p<0.05)$. Therefore, a non-parametric statistical test, Friedman test, was used to determine any differences between the three interventions existed. Level of significance was set at 0.05 . A two-tailed, Wilcoxon-signed rank test was then used to determine the differences between the three interventions with the level of significance set at $p=0.0167(p=0.05 / 3)$ after Bonferroni correction. 


\section{Results}

Twenty one participants completed the study. Table 2 provides a summary of the data collected for the twenty-one participants that took part in the trial.

The Friedman test of statistical significance showed that there was a significant difference in peak torque between the three interventions $(p=0.003)$. The results of a two-tailed, Wilcoxon-signed rank tests, with the level of significance set at 0.0167 , demonstrated that elastic therapeutic tape significantly improved peak torque when compared to the no-tape control $(p=0.005)$, whilst the differences between elastic therapeutic tape and the placebo failed to reach statistical significance $(p=0.245)$. Additionally, no significant differences were observed in terms of peak torque between the placebo taping condition and control $(p=0.245)$.

The Friedman test of statistical significance identified that there were no statistical differences between the three interventions in terms of time to peak torque $(p=0.996)$. Post-hoc analysis identified a statistical power of 0.23 , indicating that a sample size of 103 participants would be required in order to confirm the null hypothesis at a power level of 0.8 (Faul, et al., 2009).

The Friedman test order of statistical significance identified that there were no statistical differences between the three interventions in terms of peak velocity $(p=0.33)$. Post-hoc analysis identified a statistical power of 0.06 , indicating that a sample size of 1000 participants would be required in to confirm the null hypothesis at a power level of 0.8 (Faul, et al., 2009). 


\section{Discussion}

The current study aimed to investigate the effect of elastic therapeutic tape on the isokinetic and isotonic muscle performance of the lumbar extensors in healthy, asymptomatic adults. The results show that immediately following the application of elastic therapeutic tape, statistically significant improvements in peak concentric lumbar extension torque were produced when compared to a no-tape control $(p=0.005)$. This $10.52 \%$ improvement in concentric lumbar extension peak torque could also be considered to be clinically significant. This corroborated with the findings of Fratocchi et al. (2013), whereby significant improvements in peak elbow torque were recorded following the application of elastic therapeutic tape overlaying the biceps brachii muscle in comparison to a no tape control.

Contrastingly, other researchers have dismissed this association between elastic therapeutic tape and improved peak torque when incorporating alternative muscle groups such as the quadriceps and hamstrings (Fu, et al., 2008; Poon, et al., 2015; Vithoulka, et al., 2010; Wong, et al., 2012). Key differences in study design may account for these opposing findings. Whilst these studies (Fu, et al., 2008; Poon, et al., 2015; Vithoulka, et al., 2010; Wong, et al., 2012) analyzed the mean value of several peak recordings, the present study and Fratocchi et al. (2013) both investigated the best out of the attempts instead of the mean value of the peak recordings. It may be considered that the effects of elastic therapeutic tape are most apparent in conditions whereby muscles are functioning at their absolute maximal capacity and therefore when analysing mean values, differences in recordings may diminish. 
Although elastic therapeutic tape yielded improvements in peak torque when compared to the placebo taping technique, the difference was not statistically significant $(p=0.245)$. A post-hoc power analysis of a type II error of 0.86 (Power $=$ 0.14 ) indicating that a sample size of 351 would be required in order to confirm this null hypothesis at a power level of 0.8 (Faul, et al., 2007). It has been proposed that elastic therapeutic tape can enhance the neuromuscular response due to an increase in afferent stimulation of cutaneous receptors overlaying the musculature(Kase, et al., 2003; Macdonald, 2004; Macgregor, et al., 2005). Although the present study did not aim to determine the physiological processes that occur following the application of elastic therapeutic tape, it must be recognized that a placebo taping technique may provide a similar tactile stimulation to the elastic therapeutic tape overlaying the same area but failed to produce statistically significant improvements in isokinetic muscle performance. It may be possible that specific characteristics of the elastic therapeutic tape provide enhanced tactile stimulation, which in turn enhances the neuromuscular response.

Our study demonstrated there were no significant differences between the three testing conditions in terms of the time taken to reach peak torque $(p=0.996)$ and peak velocity $(p=0.33)$. However, post-hoc analysis identified statistical powers of $23.0 \%$ and $6.4 \%$ respectively. Such low statistical power signifies that in order to accept the null hypothesis, a larger sample size is required.

Wong et al. (2012) identified significant decreases in the time taken to reach peak knee extension torque following the application of elastic therapeutic tape overlaying the quadriceps muscles in healthy asymptomatic adults. Similarly, a study by Huang et al. (2007) demonstrated an earlier occurrence of vastus medialils obliquus muscle activity following the application of elastic therapeutic tape. These 
observations could be due to an increase in motor unit recruitment rate following the application of elastic therapeutic tape when compared to a no-tape control. Our study did not determine any differences between different taping conditions, a difference in the lumbar extensor muscle fibre composition and anatomical location may account for the contrasting findings when compared to studies incorporating the quadriceps.

While a previous study has validated the procedure in testing maximum velocity as a reliable method for the assessment of peak isotonic velocity of the ankle dorsiflexors and plantarflexors(Webber \& Porter, 2010), no previous studies have investigated the effect that elastic therapeutic tape has on lumbar extensor peak angular velocity. Throughout our study, a number of participants displayed apprehension towards the testing procedure due to the nature of the required highvelocity lumbar extension movement. With this in mind, it is likely that participants failed to produce the maximal results representative of their peak lumbar extension velocity, potentially producing invalid recordings. In future studies, a more functional analysis in sports performance, such as rowing, wall climbing, sprinting, rugby scrummaging and powerlifting, may be a more suitable method of measuring the effect of elastic therapeutic tape upon maximal velocity.

Although the results of this study supports the use of elastic therapeutic tape overlaying the lumbar extensors as a means of improving peak concentric torque, no evidence has investigated whether these benefits would enhance athletic performance. Previous study demonstrated significant positive correlations between isokinetic trunk strength and athletic performance in field measurements of power amongst healthy elite level tennis players (Roetert, et al., 1996). The results of this study indicate that the associated improvements in peak torque following the 
application of elastic therapeutic tape may translate in to enhanced athletic performance, however due to a paucity of research, further investigation is warranted(Roetert, et al., 1996).

The present study exhibited many strengths contributing towards the reliability of findings. By ensuring that assessors were blinded with regards to taping conditions, a lack of bias can be recognized concerning the testing procedure. Furthermore, standardization of scripted encouragement prevented a further potential risk of observer bias. Throughout the study participants were blinded with regards to whether they were receiving the elastic therapeutic tape or placebo taping condition. However, as the study did not investigate previous participant elastic therapeutic tape usage and knowledge, results may have been affected as we cannot be sure that participants were unaware of their taping condition based upon cutaneous feedback.

Concerning the instruments used within the study, isokinetic dynamometry has been validated as a highly reliable measure of peak torque and the "gold standard' for isokinetic muscle testing(Stark, et al., 2011). Although presenting a method that is clinically feasible, the application of taping conditions relies upon researcher capabilities and is subject to potential human error. In an attempt to overcome this, a single researcher applied the tape throughout the entire study. The researcher was selected based upon previous experience of elastic therapeutic tape usage and performance of tape application during the pilot study. Tape tension was determined using anthropometric measurements alongside a mathematical equation. Whilst this represents a clinically viable procedure, it cannot be guaranteed that tape tension was consistently identical for all participants. It can be suggested than in order to improve reliability, a strain gauge could be used. However, Vercelli et al. 
(2012) identified no significant changes in quadriceps muscle strength following the application of elastic therapeutic tape at various tensions, implying that tape tension does not correlate with improvements in muscle function (Vercelli, et al., 2012).

Despite participant blinding, it was impossible to conceal whether participants were receiving the no-tape control condition. This introduces a risk for performance bias within the study, whereby participants may have responded differently as they knowingly were testing the controlled variable(Pannucci \& Wilkins, 2010). This may have contributed towards the statistical differences observed between elastic therapeutic tape and the control intervention, however due to the non-significant changes observed between the placebo and control, this seems unlikely. There were a number of non-significant differences between the three testing conditions in this study. Due to a substantial type II error, further study with a larger sample size will need to be conducted before a conclusion could be made on the non-significant differences between the testing conditions.

\section{Conclusion}

The results of this study show that following the application of elastic therapeutic tape, peak lumbar extensor torque significantly improves $(p=0.005)$. No statistically significant differences in time to peak torque and peak lumber extension velocity were observed throughout the trial. However, it is not possible to make conclusions regarding peak velocity due to the potentially invalid results. Based upon these results, the application of elastic therapeutic tape overlaying the primary lumbar extensor muscles to improve peak concentric torque can be justified, 
although more research is required in order determine whether these improvements translate in to enhance athletic performance. 
Highlights

- Elastic therapeutic taping significantly improves lumbar extensor peak torque.

- Elastic taping did not improve time to peak torque during lumbar extension.

- Elastic taping did not improve peak lumbar extension velocity. 


\section{References}

1. Aktas, G., \& Baltaci, G. (2011). Does kinesio taping increase knee muscle strength and functional performance? . Isokinetics and Exercise Science, 19, 149-155.

2. De Ridder, E.M., Van Oosterwijck, J.O., Vleeming, A., Vanderstraeten, G.G., \& Danneels, L.A. (2015). Muscle functional MRI analysis of trunk muscle recruitment during extension exercises in asymptomatic individuals. Scandinavian Journal of Medicine and Science in Sports, 25, 196-204.

3. Drouin, J.M., Valovich-mcLeod, T.C., Shultz, S.J., Gansneder, B.M., \& Perrin, D.H. (2004a). Reliability and validity of the Biodex system 3 pro isokinetic dynamometer velocity, torque and position measurements. European Journal of Applied Physiology, 91, 22-29.

4. Drouin, J.M., Valovich-mcLeod, T.C., Shultz, S.J., Gansneder, B.M., \& Perrin, D.H. (2004b). Reliability and validity of the Biodex system 3 pro isokinetic dynamometer velocity, torque and position measurements. European Journal of Applied Physiology, 91, 22-29.

5. Faul, F., Erdfelder, E., Buchner, A., \& Lang, A. (2009). Statistical power analyses using G*Power 3.1: tests for correlation and regression analyses. Behavior Research Methods, 41, 1149-1160

6. Faul, F., Erdfelder, E., Lang, A.G., \& Buchner, A. (2007). G*Power 3: a flexible statistical power analysis program for the social, behavioral, and biomedical sciences. Behavior Research Methods, 39, 175-191.

7. Fratocchi, G., Di Mattia, F., Rossi, R., Mangone, M., Santilli, V., \& Paoloni, M. (2013). Influence of Kinesio Taping applied over biceps brachii on isokinetic 
elbow peak torque. A placebo controlled study in a population of young healthy subjects. Journal of Science and Medicine in Sport, 16, 245-249.

8. Fu, T.C., Wong, A.M., Pei, Y.C., Wu, K.P., Chou, S.W., \& Lin, Y.C. (2008). Effect of Kinesio taping on muscle strength in athletes-a pilot study. Journal of Science and Medicine in Sport, 11, 198-201.

9. Hicks, C.M. (2004). Research Methods for Clinical Therapists: Applied Project Design and Analysis: Churchill Livingstone \%@ 0443074305 \%7 4.

10.Hsu, Y.H., Chen, W.Y., Lin, H.C., Wang, W.T., \& Shih, Y.F. (2009). The effects of taping on scapular kinematics and muscle performance in baseball players with shoulder impingement syndrome. Journal of Electromyography and Kinesiology, 19, 1092-1099.

11. Huang, C.Y., Hsieh, T.H., Lu, S.C., \& Su, F.C. (2011). Effect of the Kinesio tape to muscle activity and vertical jump performance in healthy inactive people. Biomed Eng Online, 10, 70.

12. Imai, A., Kaneoka, K., Okubo, Y., \& Shiraki, H. (2014). Effects of two types of trunk exercises on balance and athletic performance in youth soccer players. International Journal of Sports Physical Therapy, 9, 47-57.

13. Kase, K., Wallis, J., \& Kase, T. (2003). Clinical therapeutic applications of the kinesio taping method (2nd ed.).

14.Lins, C.A., Neto, F.L., Amorim, A.B., Macedo Lde, B., \& Brasileiro, J.S. (2013). Kinesio Taping $((\mathrm{R}))$ does not alter neuromuscular performance of femoral quadriceps or lower limb function in healthy subjects: randomized, blind, controlled, clinical trial. Manual Therapy, 18, 41-45.

15. Macdonald, R. (2004). Taping techniques : principles and practice. In (2nd ed.). Edinburgh ; New York: Butterworth-Heinemann,. 
16. Macgregor, K., Gerlach, S., Mellor, R., \& Hodges, P.W. (2005). Cutaneous stimulation from patella tape causes a differential increase in vasti muscle activity in people with patellofemoral pain. Journal of Orthopaedic Research, 23, 351-358.

17. Mostert-Wentzel, K., Swart, J.J., Masenyetse, L.J., Sihlali, B.H., Cilliers, R., Clarke, L., Maritz, J., Prinsloo, E.M., \& Steenkamp, L. (2012). Effect of kinesio taping on explosive muscle power of gluteus maximus of male athletes. South African Journal of Sports Medicine, 24, 75-80.

18. Mueller, S., Stoll, J., Mueller, J., \& Mayer, F. (2012). Validity of isokinetic trunk measurements with respect to healthy adults, athletes and low back pain patients. Isokinetics and Exercise Science, 20, 255-266.

19. Newton, R.U., \& Kraemer, W.J. (1994). Developing explosive muscular power: Implication for a mixed methods training strategy. Journal of Strength and Conditioning Research, 16, 20-31.

20.Pannucci, C.J., \& Wilkins, E.G. (2010). Identifying and avoiding bias in research. Plastic and Reconstructive Surgery, 126, 619-625.

21.Polgar, S., \& Thomas, S.A. (2008). Introduction to research in the health sciences. In (5th ed.). Edinburgh: Elsevier,.

22.Poon, K.Y., Li, S.M., Roper, M.G., Wong, M.K., Wong, O., \& Cheung, R.T. (2015). Kinesiology tape does not facilitate muscle performance: A deceptive controlled trial. Manual Therapy, 20, 130-133.

23. Roetert, E.P., McCormick, T.J., Brown, S.W., \& Ellenbecker, T.S. (1996). Relationship between isokinetic and functional trunk strength in elite junior tennis players. Isokinetics and Exercise Science, 6, 15-20. 
24.Sharp, T., Halaki, M., Greene, A., \& Vanwanseele, B. (2014). An EMG assessment of Front Row Rugby Union Scrummaging. International Journal of Performance Analysis in Sport, 14, 225-237.

25. Slupik, A., Dwornik, M., Bialoszewski, D., \& Zych, E. (2007). Effect of Kinesio Taping on bioelectrical activity of vastus medialis muscle. Preliminary report. Ortop Traumatol Rehabil, 9, 644-651.

26. Stark, T., Walker, B., Phillips, J.K., Fejer, R., \& Beck, R. (2011). Hand-held dynamometry correlation with the gold standard isokinetic dynamometry: a systematic review. Pm r, 3, 472-479.

27. Vercelli, S., Sartorio, F., Foti, C., Colletto, L., Virton, D., Ronconi, G., \& Ferriero, G. (2012). Immediate effects of kinesiotaping on quadriceps muscle strength: a single-blind, placebo-controlled crossover trial. Clinical Journal of Sport Medicine, 22, 319-326.

28. Vithoulka, I., Beneka, P., Malliou, A., Aggelousis, N., Karatsolis, K., \& Diamantopoulos, K. (2010). The effects of kinesio-taping on quadriceps strength during isokinetic exercise in healthy non athlete women. Isokinetics and Exercise Science, 18, 1-6.

29. Webber, S.C., \& Porter, M.M. (2010). Reliability of ankle isometric, isotonic, and isokinetic strength and power testing in older women. Physical Therapy, $90,1165-1175$.

30. Wilmore, J.H., Costill, D.L., \& Kenney, W.L. (2008). Physiology of sport and exercise. In (4th ed.). Champaign, III.: Human Kinetics,.

31. Wong, O.M., Cheung, R.T., \& Li, R.C. (2012). Isokinetic knee function in healthy subjects with and without Kinesio taping. Physical Therapy in Sport 13, 255-258. 


\section{List of figures.}

Figure 1. Standardized taping technique

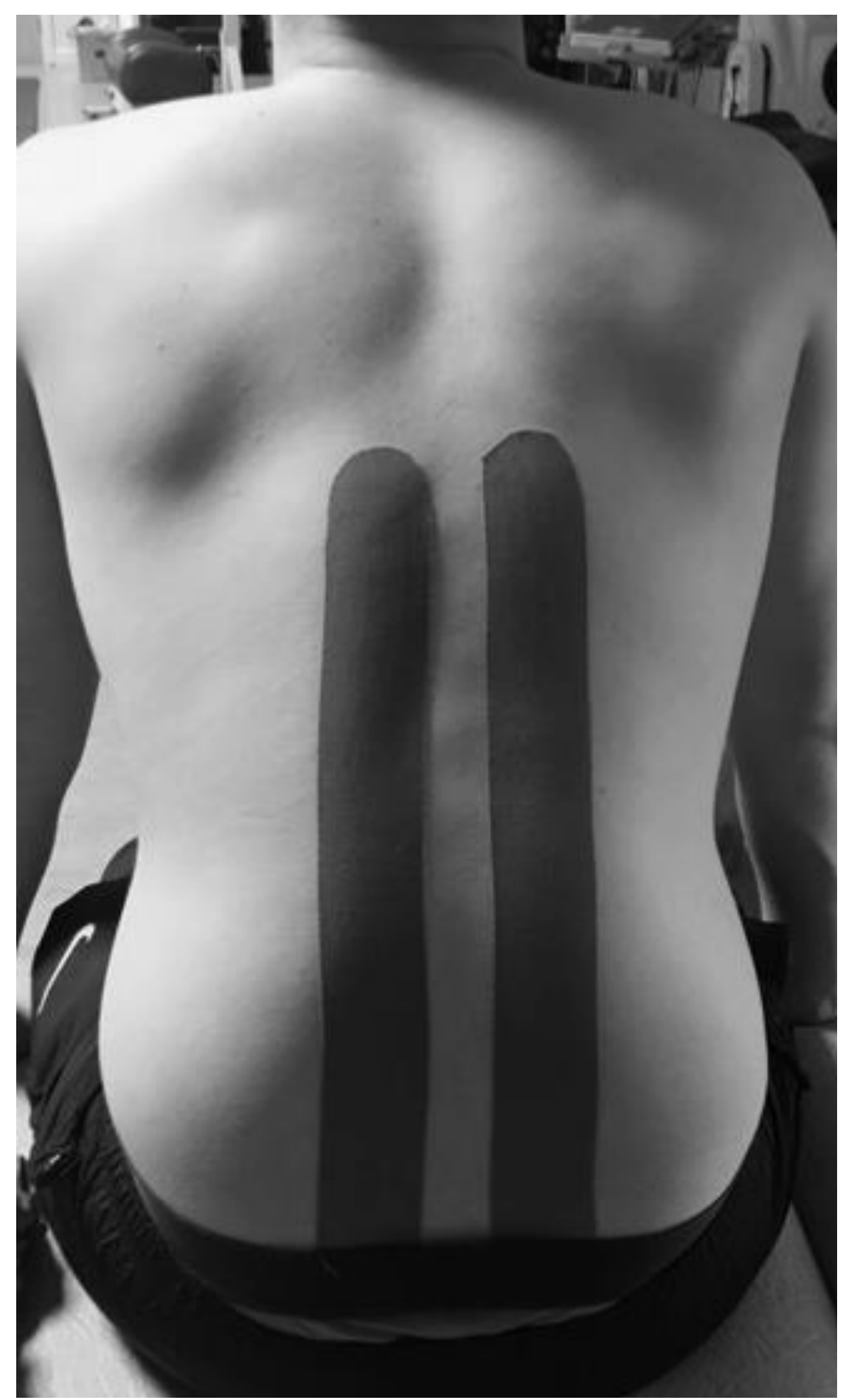


Figure 2. Standardized isokinetic dynamometer seating position

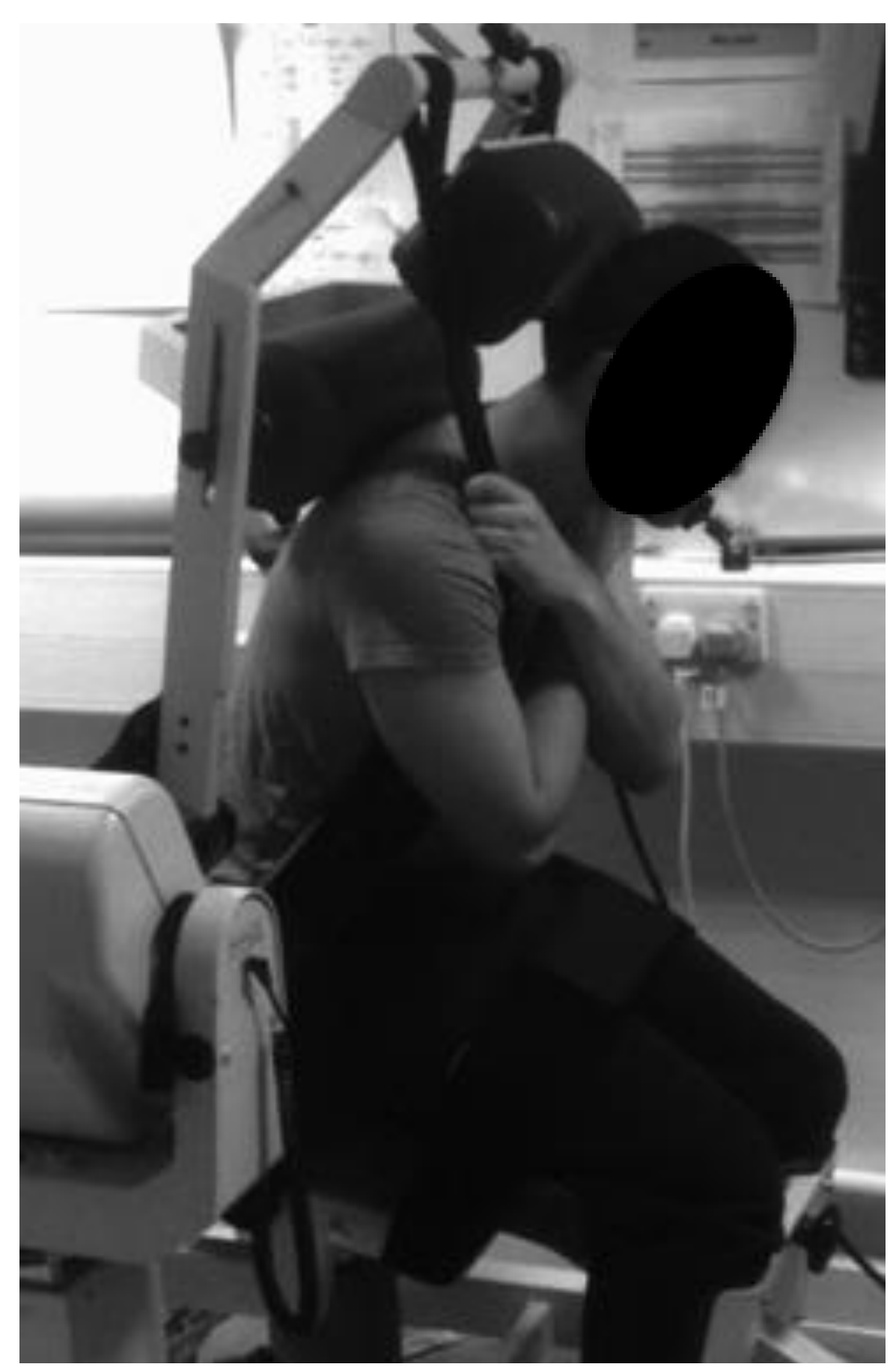

\section{List of tables.}


Table 1: Demographics of the participants

Participants $(n=21)$

Age in years, mean \pm standard deviation

$23.62 \pm 4.58$

Sex

Male, n (\%)

$11(52.4)$

Female, $\mathrm{n}(\%)$

$10(47.6)$

Height $(\mathrm{cm})$, mean \pm standard deviation

$171.76 \pm 6.64$ 


Mean \pm
standard
deviation Median $\quad \begin{gathered}25^{\text {th }} \\ \text { percentile }\end{gathered} \begin{gathered}75^{\text {th }} \\ \text { percentile }\end{gathered}$

Concentric lumbar extension peak torque $(\mathrm{N})$

\begin{tabular}{lllll}
\hline Elastic therapeutic tape & $282.44 \pm 69.29$ & 298.54 & 221.81 & 345.86 \\
\hline Placebo tape & $276.01 \pm 73.54$ & 290.37 & 207.01 & 345.23 \\
\hline No tape & $272.86 \pm 68.43$ & $270.12^{*}$ & 201.15 & 342.89 \\
\hline
\end{tabular}

\begin{tabular}{lcccc}
\hline Time to peak torque $(\mathrm{s})$ & & & & \\
\hline Elastic therapeutic tape & $0.18 \pm 0.12$ & 0.14 & 0.125 & 0.17 \\
\hline Placebo tape & $0.19 \pm 0.22$ & 0.14 & 0.13 & 0.165 \\
\hline No tape & $0.26 \pm 0.32$ & 0.15 & 0.12 & 0.175
\end{tabular}

\begin{tabular}{lllll}
\hline Peak velocity $\left(\mathrm{m} \mathrm{s}^{-1}\right)$ & & & \\
\hline Elastic therapeutic tape & $230.78 \pm 33.71$ & 247.87 & 211.19 & 252.54 \\
\hline Placebo tape & $233.75 \pm 41.61$ & 246.68 & 216.97 & 252.31 \\
\hline No tape & $227.62 \pm 35.83$ & 244.68 & 212.08 & 252.67
\end{tabular}

${ }^{*} p<0.0167$, significant difference in the concentric lumbar extension peak torque (N) between elastic therapeutic tape and no tape. 